\title{
MALIGNANT NEOPLASMS OF BONE AND ARTICULAR CARTILAGE -HOSPITAL BURDEN IN ROMANIA
}

\author{
doi: 10.2478/rojost-2018-0054 \\ D.G. Mincă ${ }^{1}$, C. Cirstoivi ${ }^{1}$ F.L. Furtunescu ${ }^{1}$, R.V. Costea ${ }^{1}$ \\ "Carol Davila" University of Medicine and Pharmacy, Bucharest, Romania
}

Introduction. Cancers of bone and articular cartilage are relatively rare, and a global analysis was not performed up to present in Romania due to the lack of a national registry for these diseases. This study aimed to explore the hospital burden due to malignant neoplasm of bone and articular cartilage in Romania and the general characteristics of the hospitalized cases.

Materials and methods. We used the data reported in the routine statistic system during 2012 and 2016 and we analyzed the number of cases discharged from hospitals by age group, gender, and duration of hospitalization.

Results. The number of hospitalized cancers of bone and articular cartilages decreased by $17 \%$ in the last five years (2012-2016) but this decrease was higher in other/ unspecified cancers (22\%) and lower in limb cancers (10\%).

Among the 1872 cases reported in 2016, 47\% were limbs' cancers and the rest, cancers of others/ unspecified sites. Males accounted for $62 \%$ of all cancers ( $58 \%$ of limbs cancers and $65 \%$ of other cancers, $\mathrm{p}=0.001, \mathrm{Chi}^{2}$ test). Distribution by age showed that $17 \%, 62 \%$ and $21 \%$ of the cancers occurred in age-groups $0-14,15-64$ and $65+$ respectively, but limbs cancers occurred in a significantly higher proportion in children ( $29 \%$ vs. $7 \%$ of other cancers, $\mathrm{p}<0.001$ ). 16983 days of hospitalization were reported in 2016 for this pathology, with an average length of stay of 9.07 days (9.37 and 8.81 days in limbs and other cancers respectively).

Conclusions. More detailed analysis of routine reported data is required for understanding the characteristics and trends of bone cancers in Romania.

Keywords: malignant neoplasm of bone and articular cartilage, hospital burden, trends 